\title{
Virtual simulation technology in the shooting motion
}

\author{
Shaojian Li \\ College of Art, TongHua Normal University, Jilin 134002, China \\ 102212189@qq.com
}

Keywords: Motion simulation; Virtual basketball shooting; New technology.

\begin{abstract}
This paper, using Motion simulation in virtual basketball shooting process, simulates the process of moving to basketball players to provide scientific reference data. With basketball shooting of new technologies and methods emerging, people should research and summarize its methods and characteristics of the understanding and mastering the principle, while requires people have profound recognition Knowledge and understanding to new technology and new methods , in order to keep up with the development of the technology.
\end{abstract}

\section{Introduction}

In basketball, shooting is the only means of getting scores, which is the focus of the game, as well as the core of controlling and anti-controlling for two sides, in order to make victory in the race, it should improve the hit rate of shooting, they are only asked to put emphasis on the action or simply imitate the demonstration action; by using such mechanical training and mechanical form, it is easy for the students to make all kinds of wrong action without knowing it, when the problem is found, it has wasted a lot of time, it needs more time and energy to correct the mistakes, which results in the low efficiency of training and learning, therefore, it should explore a method for improving the efficiency of the shooting training in basketball shooting training, therefore, using virtual simulation technology in the training of basketball shooting is the purpose of this thesis

\section{Ball Animation Algorithm of Virtual Shooting}

This section studies ball's motion animation in the large scale scene. Spherical object is a kind of basic geometric object, with broad representation. Here mainly considered for virtual shooting system, studying the elastic deformation of the spherical surface and parabolic motion animation of spherical object. Elastic deformation is a common pattern of deformation, after the object occurred the deformation, it can restore the original shape according to the elastic model.

The algorithm of elastic deformation is based on particle system, at the same time, gridless method is adopted to improve the efficiency of the calculation of deformation. It will make each vertex of the dimensional objects that formed the three-dimensional deformation as particle, thus, the deformed object can represent a particle system, by controlling the particle, it can also change the shape of object. At the same time, it sets up a corresponding target position for each particle that consisted of the deformation objects.

After occurring the deformation, elastic power will pull the particles to the target position, so that the deformed object can restore the original shape, the object in the thesis that is dealt with deformed algorithm is a three-dimensional object composed by the particle with quality and initial position. Because there is no connected information between each particles, so it need not consider the interaction between particles, it only need consider the collision of particle and environment, as well as the external force of the particle.It is assumed that the corresponding relationship is known between the initial shape and the actual shape of the object.

Therefore, the main task is to determine the optimal rigid transformation of the two point clouds, the problem can be stated as follows: for the two groups of given point: $\mathrm{xi}$ and $\mathrm{x} 0$, calculate the rotation matrix $\mathrm{R}$ and the translation vector $\mathrm{t}$ and $\mathrm{t} 0$, minimizing the formula below: 


$$
\sum_{i} w_{i}\left(R\left(x_{i}^{0}-t_{0}\right)+t-x_{i}\right)^{2}
$$

Among them, mi is the weight value of each point, apparently the optimal translation vector is the centroid of the initial shape and the actual shape, i.e.:

$$
t_{0}=x_{c m}^{0}=\frac{\sum_{i} m_{i} x_{i}^{0}}{\sum_{i} m_{i}}, t=x_{c m}=\frac{\sum_{i} m_{i} x_{i}}{\sum_{i} m_{i}}
$$

Among them, Tvv is a symmetric matrix, containing only scaling without rotation. Therefore, the optimum rotary torque array $\mathrm{R}$ is the rotating part of matrix Tuv, through the polar decomposition $T_{u v}=R \cdot S$, R can be obtained, among them, $S=\sqrt{T_{u v}^{T} T_{u v}}$ is the symmetric part, $R=T_{U V} S^{-1}$ is the rotating part, the final target position can be calculated by the following formula:

$$
g_{i}=R\left(x_{i}^{0}-x_{c m}^{0}\right)+x_{c m}
$$

Among them, a is the parameter of object's hardness, fext is the external force on the particle. According to the algorithm of elastic deformation, it can realize the simulation of object's deformation.

$$
\begin{aligned}
& v_{i}=(t+\Delta t)=v_{i}(t)+a \frac{g_{i}(t)-x_{i(t)}}{\Delta t}+\Delta t \cdot f_{e x t}(t) / m_{i} \\
& x_{i}(t+\Delta t)=x_{i}(t)+\Delta t \cdot v_{i}(t+\Delta t)
\end{aligned}
$$

\section{Check the Algorithm of Elastic Deformation}

The deformed object used here is the $3 \mathrm{D}$ model of basketball, from the left to the right is the process of the ball falling from the sky to the ground, collided and deformed, bounced back to the air, and restored to the original shape. By using the algorithm of elastic deformation proposed in this thesis to simulate the deformation of basketball, after the deformation of the sphere, it can be recovered to the original shape.

The following described the performance of the algorithm of elastic deformation, it can be seen, as the number of particles of the deformed object is increased, the rendering speed is decreased, this is because with the increased size of the particles, it requires more resources to calculate the deformation; but compared with the calculation of not adopting deformation algorithm, adopting the algorithm of deformation does not make the rendering speed dropped a lot, this because in this thesis it adopts gridless method, which dose not need to consider, the interaction between the particles, thus, it reduces the complexity and improves the efficiency of calculation.

Therefore, the algorithm of elastic deformation can be enough to meet the need of real-time rendering. As shown in table 1.

Tab. 1 the Performance of Algorithm of Elastic Deformation

\begin{tabular}{|c|c|c|c|}
\hline $\begin{array}{c}\text { Rendering frames } \\
\text { The number of particles }\end{array}$ & 2710 & 3050 & 3745 \\
\hline The algorithm without deformation algorithm & $59 F P S$ & $52 F P S$ & $45 F P S$ \\
\hline The algorithm added with deformation algorithm & $49 F P S$ & $43 F P S$ & $37 F P S$ \\
\hline
\end{tabular}

According to the analysis of virtual shooting of basketball. First of all, basketball games are usually held indoors, so there is no interference of wind and rain. The influence of the power from the 
gravity and air resistance in the process of basketball's rapid flight is far greater than the effect of buoyancy of the air and air pressure. Therefore, it can ignore the influence factors of air buoyancy and air pressure. Secondly, the moving distance of basketball is much smaller than the radius of the earth. So, it can be assumed that the surface of the earth is flat rather than spherical and it can ignore the Coriolis inertial force caused by the rotation of the earth. Finally, assuming that the shape of basketball is in the spherical shape with standard axial symmetry during the flight, the effect of the air friction on the speed of basketball can also be ignored.

The motion model proposed in this thesis is mainly considering the effect of gravity and the air resistance on basketball. Through analyzing the force of gravity and the air resistance, the flight trajectory of basketball is a parabola.

The parabola trajectory of basketball and the final landing point is depended on the initial velocity vector that fingers of athletes left basketball, including velocity and direction. Using $\mathrm{X}^{\prime}, \mathrm{Y}$ and $\mathrm{Z}$ as $3 \mathrm{D}$ coordinate to describe the motion model of basketball.

The $\mathrm{X}^{\prime}$ axis represents the horizontal direction from hand to basketball rack, Y axis represents the moving height of basketball. It can be assumed that the deflexion-angle of $X^{\prime}$ axis and the direction of the actual shooting in $\mathrm{X} Z \mathrm{Z}$ plane is a.

Assuming $\mathrm{Xk}$ axis is the projection of basketball's flying parabolic path in $\mathrm{X} Z \mathrm{Z}$ plane. The following is the analysis on $\mathrm{XkY}$, which is as shown in Fig. 1:

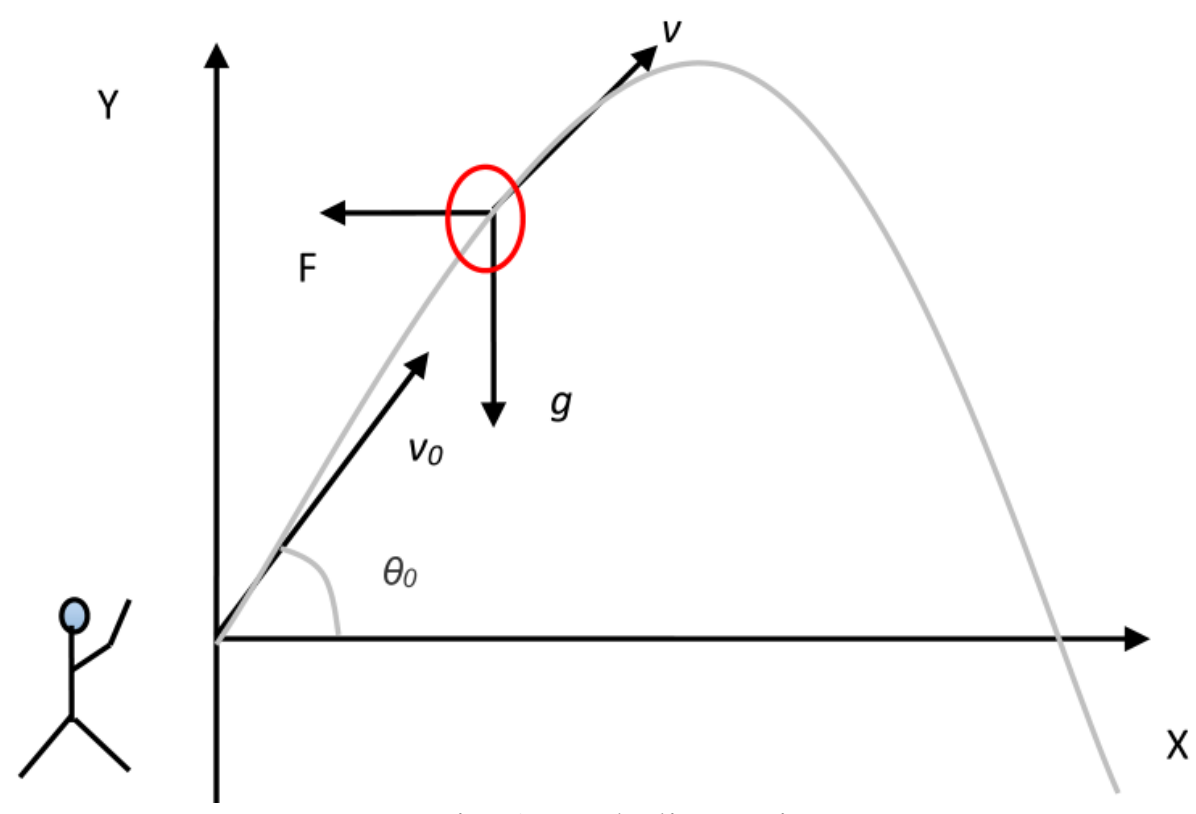

Fig. 1 Parabolic Motion

Virtual 3D shooting regards the shooting as an independent and complete system, using the information feedback function of the system to correct errors in each exercise timely, which can make the action more and more reasonable, and the system can evaluate from the initial disordered state to the ordered state, so as to reach a new equilibrium state, namely the action can form the dynamic stereotype. In the evolution process, the exercisers are always controlling the technique shooting action, so as to the shooting action can be completed according to the set procedures.

\section{Conclusion}

This thesis proposed the model of parabolic motion focused on basketball sports, realizing the animation of basketball after being thrown in the air. In the future work, teachers should combine the model of parabolic motion and the algorithm of elastic deformation to simulate various possible real shooting situations during the process of shooting, realizing the real simulation of basketball. 


\section{Reference}

[1] Wang, H.G., The principle and application of computer simulation. National Defense University of science and technology press. (2004).

[2] Williamson, O., Computer simulation technology applied in the field of sports training. Sports research. 68-70 (2008).

[3] Luo. X. D., Virtual reality technology and its application in training. Education technology. 53-54 (2003).

[4] Rajan, R., 3D face modeling method based on plane surface. Computer engineering and design, 157-159(2009). 